\begin{tabular}{|c|c|c|c|c|c|c|c|c|}
\hline values (mean) & $\begin{array}{l}\text { Baseline LH/ } \\
\text { FSH ( }\end{array}$ & $\begin{array}{l}\text { Follow up } \\
\text { LH/FSH }\end{array}$ & $\begin{array}{l}\text { Baseline } \\
\text { E2 }\end{array}$ & $\begin{array}{l}\text { Follow up } \\
\text { E2 }\end{array}$ & $\begin{array}{l}\text { Baseline AMH/ } \\
\text { Inhibin B }\end{array}$ & $\begin{array}{l}\text { Follow up AMH/ } \\
\text { Inhibin B }\end{array}$ & $\begin{array}{l}\text { Baseline Follicular count/ } \\
\text { dominant follicle }\end{array}$ & $\begin{array}{l}\text { Follow up Follicular count/ } \\
\text { dominant follicle }\end{array}$ \\
\hline Cases $n=25$ & $5.82 / 5.23$ & $17.6 / 25.08$ & 80.156 & 56.063 & $5.667 / 56.11$ & $1.223 / 16.48$ & $4 / 1$ & $1 / 0.5$ \\
\hline$p$ value & $0.14 / 0.043$ & & 0.13 & & $0.003 / 0.001$ & & 0.001 & \\
\hline Controls $n=25$ & $9.41 / 4.78$ & $8.28 / 6.1$ & 89.622 & 112.9 & $6.3 / 53.07$ & $5.78 / 82.87$ & $3.5 / 1.1$ & $4 / 1$ \\
\hline$p$ value & $0.585 / 0.087$ & & 0.310 & & $0.745 / 0.001$ & & $>0.05$ & \\
\hline $\begin{array}{l}\text { Comparison cases- controls } \\
\text { p value }\end{array}$ & $0.065 / 0.938$ & $0.554 / 0.357$ & 0.749 & 0.002 & $0.061 / 0.547$ & $0.001 / 0.001$ & $>0.05$ & 0.001 \\
\hline
\end{tabular}

\section{INDUCTION AND MAINTENANCE TREATMENT OF PROLIFERATIVE LUPUS NEPHRITIS: AN UPDATED COCHRANE REVIEW}

\begin{abstract}
1,2 DJ Tunnicliffe ${ }^{*},{ }^{3} \mathrm{SC}$ Palmer, ${ }^{1,2} \mathrm{JC}$ Craig, ${ }^{1,2,4} \mathrm{AC}$ Webster, ${ }^{5} \mathrm{LK}$ Henderson, ${ }^{1,2,6 \mathrm{P}}$ Masson, ${ }^{1,2} \mathrm{~A}$ Tong, ${ }^{7,8,9} \mathrm{D}$ Singh-Grewal, ${ }^{10} \mathrm{R}$ Flanc, ${ }^{11} \mathrm{MA}$ Roberts, ${ }^{1,2,12,13} \mathrm{GFM}$. Strippoli. ${ }^{1}$ University of Sydney, Sydney School of Public Health, Sydney, Australia; ${ }^{2}$ Children's Hospital at Westmead, Centre for Kidney Research, Sydney, Australia; ${ }^{3}$ University of Otago, Department of Medicine, Christchurch, New Zealand; ${ }^{4}$ Westmead Institute, Centre for Transplant and Renal Research, Sydney, Australia; ${ }^{5}$ Royal Infirmary of Edinburgh, Department of Renal Medicine, Edinburgh, UK; ${ }^{6}$ University of Edinburgh, Department of Medicine, University of Edinburgh, UK; ' University of Sydney, Sydney Medical School, Sydney, Australia; ${ }^{8}$ The University of New South Wales, Faculty of Medicine, Sydney, Australia; ${ }^{9}$ Sydney Children's Hospital Network, Department of Rheumatology, Sydney, Australia; ${ }^{10}$ Monash Medical Centre, Department of Nephrology, Melbourne, Australia; ${ }^{11}$ Eastern Health Clinical SchoolMonash University, Department of Nephrology, Box Hill- Australia, Australia; ${ }^{12}$ University of Bari, Department of Emergency and Organ Transplantation, Bari, Italy; ${ }^{13}$ Diaverum, Medical Scientific Office, Lund, Sweden
\end{abstract}

\subsection{6/lupus-2017-000215.254}

Background and aims Pharmacological treatments have improved survival in lupus nephritis. However, intravenous cyclophosphamide as first-line therapy has considerable toxicity and lacks evidence of efficacy to prevent end-stage kidney disease. The comparative efficacy of newer strategies compared with intravenous cyclophosphamide remains unclear.
Methods We updated a random-effects meta-analysis of randomised controlled trials on induction and maintenance therapy for proliferative lupus nephritis. Evidence quality was assessed using GRADE.

Results 59 trials (4465 participants) were eligible, including nine new trials. Compared with intravenous cyclophosphamide, mycophenolate mofetil (MMF) incurred similar risks of complete remission, mortality, or major infection, while risks of alopecia and ovarian failure were lower (Table 1) (evidence quality=moderate). There was no evidence combined MMF and tacrolimus had different effects on complete remission or major infection than intravenous cyclophosphamide (Table 1 ) (evidence quality =low-very low). In maintenance therapy (Table 2), MMF decreased risks of disease relapse compared to azathioprine (evidence quality=moderate), although there was no evidence of different effects between maintenance therapies on mortality, end-stage kidney disease, or major infection (evidence quality $=$ very low - low).

Conclusions MMF is as effective as intravenous cyclophosphamide in inducing remission in patients with proliferative lupus nephritis, with lower risks of alopecia and ovarian failure, although comparative effects of treatment on end-stage kidney disease and mortality remain uncertain. MMF is the most effective maintenance treatment to prevent relapse.

Abstract 254 Table 1 Summary of findings for induction therapy

\begin{tabular}{|c|c|c|c|c|c|}
\hline Intervention & Comparison & Outcome & $\begin{array}{l}\text { Number of } \\
\text { participants } \\
\text { (studies) }\end{array}$ & $\begin{array}{l}\text { Relative risk } \\
(95 \% \text { CI) }\end{array}$ & $\begin{array}{l}\text { Quality of the } \\
\text { evidence (GRADE) }\end{array}$ \\
\hline \multirow[t]{5}{*}{$\begin{array}{l}\text { Mycophenolate } \\
\text { mofeetl }\end{array}$} & $\begin{array}{l}\text { Intravenous } \\
\text { cyclophosphamide }\end{array}$ & $\begin{array}{l}\text { Complete renal } \\
\text { remission }\end{array}$ & 806 (7 studies) & $1.26(0.97,1.64)$ & Moderate \\
\hline & & Mortality & 800 (7 studies) & $1.34(0.65,2.76)$ & Moderate \\
\hline & & Major infection & 773 ( 6 studies) & $1.09(0.94,1.26)$ & Moderate \\
\hline & & Alopecia & 622 (3 studies) & $0.29(0.19,0.46)$ & Low \\
\hline & & Ovarian failure & 685 (5 studies) & $0.35(0.12,0.98)$ & Moderate \\
\hline \multirow[t]{2}{*}{\begin{tabular}{l|} 
Mycophenolate \\
moteetl t tacrolimus
\end{tabular}} & $\begin{array}{l}\text { Intravenous } \\
\text { cyclophosphamide }\end{array}$ & $\begin{array}{l}\text { Complete renal } \\
\text { remission }\end{array}$ & 402 (2 studies) & $3.23(0.64,16.45)$ & Low \\
\hline & & Major infection & 402 (2 studies) & $2.75(0.99,7.62)$ & Very low \\
\hline
\end{tabular}


Abstract 254 Table 2 Summary of findings for maintenance therapy

\begin{tabular}{|c|c|c|c|c|}
\hline Comparison & Outcome & $\begin{array}{l}\text { Number of } \\
\text { participants (studies) }\end{array}$ & Relative risk & $\begin{array}{l}\text { Quality of the } \\
\text { evidence (GRADE) }\end{array}$ \\
\hline \multirow[t]{4}{*}{ Mycophenolate moletil Azathioprine } & Renal relapse & 452 (4 studies) & $0.63(0.42,0.95)$ & Moderate \\
\hline & Mortality & 452 (4 studies) & $0.87(0.26,2.94)$ & Very low \\
\hline & $\begin{array}{l}\text { End-stage kidney } \\
\text { disease }\end{array}$ & 452 (4 studies) & $0.75(0.28,2.04)$ & Very low \\
\hline & Major infection & 412 (3 studies) & $0.92(0.51,1.67)$ & Low \\
\hline
\end{tabular}

\section{PREDICTORS OF REMISSION AND LOW LUPUS DISEASE ACTIVITY STATUS (LLDAS): DATA FROM A MULTI- ETHNIC, MULTINATIONAL LATIN AMERICAN LUPUS COHORT}

\begin{abstract}
${ }^{1,2} \mathrm{M}$ Ugarte-Gil ${ }^{*},{ }^{3} \mathrm{D}$ Wojdyla, ${ }^{4} \mathrm{G}$ Pons-Estel, ${ }^{5} \mathrm{~J}$ Gomez-Puerta, ${ }^{6} \mathrm{~L}$ Catoggio, ${ }^{7} \mathrm{~A}$ Alvarellos, ${ }^{7} \mathrm{~V}$ Saurit, ${ }^{8} \mathrm{E}$ Borba, ${ }^{9} \mathrm{E}$ Sato, ${ }^{10} \mathrm{~L}$ Costallata, ${ }^{11} \mathrm{~N}$ Da Silva, ${ }^{12} \mathrm{~A}$ Iglesias-Gamarra, ${ }^{13} \mathrm{O}$ Neira, ${ }^{14} \mathrm{G}$ Reyes-Llerena, ${ }^{15} \mathrm{M}$ Cardiel, MC Amigo ${ }^{16},{ }^{1} \mathrm{E}$ Acevedo-Vasquez, ${ }^{17} \mathrm{M}$ Esteva-Spinetti, ${ }^{18} \mathrm{G}$ Alarcón, ${ }^{19} \mathrm{~B}$ Pons-Estel. ${ }^{1}$ Hospital Guillermo Almenara Irigoyen. EsSalud, Rheumatology, Lima, Peru; ${ }^{2}$ Universidad Científica del Sur, School of Medicine, Lima, Peru; ${ }^{3}$ GLADEL, Consultant, Rosario, Argentina; ${ }^{4}$ Hospital Clinic, Department of Autoimmune Diseases, Barcelona, Spain; ${ }^{5}$ Universidad de Antioquia, Facultad de Medicina-, Antioquia, Colombia; ${ }^{6}$ Hospital Italiano, Sección de Reumatología, Buenos Aires, Argentina; ${ }^{7}$ Hospital Privado, Servicio de Reumatología, Cordoba, Argentina; ${ }^{8} \mathrm{Hospital}$ das Clínicas da Faculdade de Medicina da Universidade de São Paulo, Rheumatology, São Paulo, Brazil, ${ }^{9}$ Universidade Federal de São Paulo, Departamento de Medicina, São Paulo, Brazil; ${ }^{10}$ Universidade Estadual da Campinas, Divisao de Reumatologia- Faculdade de Ciencias Medicas, Campinas, Brazil; ${ }^{11}$ Universidade Federal de Goiás, Faculdade de Medicina, Goiânia, Brazil; ${ }^{12}$ Universidad Nacional de Colombia, Facultad de Medicina, Bogotá, Colombia; ${ }^{13}$ Universidad de Chile, Facultad de Medicina, Santiago, Chile; ${ }^{14}$ Centro de Investigaciones Médico Quirúrgicas -CIMEQ-, Reumatología, Habana, Cuba; ${ }^{15}$ Centro de Investigación Clínica de Morelia, Reumatología, Morelia, Mexico; ${ }^{16}$ Centro Medico ABC, Reumatología, México, Mexico; ${ }^{17}$ Hospital Central de San Cristóbal, Reumatología, San Cristóbal, Venezuela; ${ }^{18}$ The University of Alabama at Birmingham, Department of Medicine, Birmingham, USA; ${ }^{19}$ Hospital Provincial de Rosario, Reumatologia, Rosario, Argentina
\end{abstract}

10.1136/lupus-2017-000215.255

Background and aims Remission and LLDAS prevent the occurrence of damage accrual in SLE patients. The aim of this study was to evaluate the predictors of remission and LLDAS in SLE patients.

Methods Three disease activity statuses were defined: Remission $=\mathrm{SLEDAI}=0$ and a prednisone dose $\leq 5 \mathrm{mg} / \mathrm{d}$ and/or immunosuppressive drugs in maintenance dose; LLDAS=SLEDAI $\leq 4$, a prednisone dose $\leq 7.5 \mathrm{mg} / \mathrm{d}$ and/or immunosuppressive drugs in maintenance dose; and non-optimally controlled status $=$ SLEDAI $>4$ and $/$ or prednisone dose $>7.5 \mathrm{mg} / \mathrm{d}$ and/or IS drugs in induction dose. Antimalarials were allowed in all groups. Patients with at least two SLEDAI reported and not optimally controlled at cohort entry were included in this analysis. Predefined outcomes were remission and remission/LLDAS. Potential predictors were gender, age at diagnosis, ethnicity, socioeconomic status, residence, health insurance, disease duration at cohort entry, organs/systems affected at or before cohort entry, treatment at or before cohort entry and SLEDAI at cohort entry. Univariable and multivariable Cox regression models with a stepwise selection procedure were performed for remission alone and for remission/LLDAS.

Results One-thousand one-hundred and forty patients were non-optimally controlled at cohort entry. One hundred and ninety-six patients achieved remission (17.2\%) and 314 achieved remission/LLDAS (27.5\%). Predictors of remission and remission/LLDAS in the multivariable models are depicted in Tables 1 and 2 .

Conclusions Mucocutaneous manifestations, renal involvement and higher disease activity early in the course of SLE were

Abstract 255 Table 1 Predictors of remission. Multivariable model.

\begin{tabular}{lll}
\hline Predictor & Hazard ratio $(95 \% \mathrm{Cl})$ & p value \\
\hline $\begin{array}{ll}\text { Socioeconomic status } \\
\text { High/medium high }\end{array}$ & Ref. & \\
Medium & $0.856(0.543-1.348)$ & 0.5023 \\
Medium low/low & $0.626(0.407-0.961)$ & 0.0323 \\
Mucocutaneous involvement & $0.638(0.433-0.940)$ & 0.0230 \\
Renal involvement & $0.716(0.523-0.981)$ & 0.0373 \\
SLEDAl at baseline & $0.975(0.955-0.996)$ & 0.0188 \\
\hline
\end{tabular}

